\title{
Parietal Involvement in Constructional Apraxia as Measured Using the Pentagon Copying Task
}

\author{
Stefan Van der Stigchel ${ }^{a} \quad$ Jeroen de Bresserb, c Rutger Heinen ${ }^{d}$ \\ Huiberdina L. Koek ${ }^{e}$ Yael D. Reijmer ${ }^{d}$ Geert Jan Biessels ${ }^{d}$ \\ Esther van den Berg ${ }^{f}$ on behalf of the Utrecht Vascular Cognitive Impairment \\ (VCl) Study Group

\begin{abstract}
aExperimental Psychology, Helmholtz Institute, Utrecht University, Utrecht, The Netherlands; ${ }^{b}$ Department of Radiology, Leiden University Medical Centre, Leiden, The Netherlands; 'Department of Radiology, University Medical Centre Utrecht, Utrecht, The Netherlands; ${ }^{\mathrm{d}}$ Department of Neurology, University Medical Centre Utrecht, Utrecht, The Netherlands; e Department of Geriatrics, University Medical Centre Utrecht, Utrecht, The Netherlands; fDepartment of Neurology, Erasmus MC University Medical Centre, Rotterdam, The Netherlands
\end{abstract}

\author{
Keywords \\ Constructional apraxia $\cdot$ Neurodegenerative disease $\cdot$ Visual attention
}

\begin{abstract}
Deficits in copying ("constructional apraxia") is generally defined as a multifaceted deficit. The exact neural correlates of the different types of copying errors are unknown. To assess whether the different categories of errors on the pentagon drawing relate to different neural correlates, we examined the pentagon drawings of the MMSE in persons with subjective cognitive complaints, mild cognitive impairment, or early dementia due to Alzheimer's disease. We adopted a qualitative scoring method for the pentagon copy test (QSPT) which categorizes different possible errors in copying rather than the dichotomous categories "correct" or "incorrect." We correlated (regional) gray matter volumes with performance on the different categories of the QSPT. Results showed that the total score of the QSPT was specifically associated with parietal gray matter volume and not with frontal, temporal, and occipital gray matter volume. A more fine-grained analysis of the errors reveals that the intersection score and the number of angles share their underlying neural correlates and are associated with specific subregions of the parietal cortex. These results are in line with the idea that constructional apraxia can be attributed to the failure to integrate visual information correctly from one fixation to the next, a process called spatial remapping.

(C) 2018 The Author(s)

Published by S. Karger AG, Basel
\end{abstract}

Members of the Utrecht Vascular Cognitive Impairment Study Group are listed in the Appendix. 
Copying a drawing is a complex cognitive operation that involves various cognitive domains, including visual perception, visual imagery, and graphic production (i.e., translating the visual image to a motor command [1]). Deficits in copying can occur after stroke [2, 3], but are also observed in Alzheimer's disease (AD) [4, 5], dementia with Lewy bodies [4], and patients with mild cognitive impairment (MCI) $[5,6]$. Deficits in copying are generally referred to as "constructional apraxia," defined as an acquired deficit in copying even simple drawings accurately, without difficulties in making individual motor movements [7]. Besides difficulties in copying, constructional apraxia is also characterized by problems in building and assembling objects. Ala et al. [4] reported that $60 \%$ of patients with AD included in their study had drawing difficulties, as objectified using the Pentagon copying task of the Mini-Mental State Examination (MMSE).

The range of cognitive functions involved in copying implies that a unifying explanation for all constructional apraxia is implausible. Given that a widespread neural network is required for accurate copying [8], damage to different parts of this network could result in different symptoms on copying tasks. It has therefore been suggested that constructional apraxia should not be considered a unitary deficit, but is better defined as a multifaceted deficit that can be the result of different neural correlates [9]. It has indeed been suggested that deficits after frontal or parietal damage differ [for a review, see 10]. In this explanation, damage to the frontal regions is more related to errors in perseveration, resulting in patients producing the same figure repeatedly, and errors in planning, resulting in poor overall organization [11]. Parietal damage results in rotational errors and errors in orientation [12], due to deficits in visuospatial and selective attention. It has recently even been proposed that the key deficit in constructional apraxia is the failure to integrate visual information correctly from one eye movement fixation to the next, a crucial function of the posterior parietal cortex [3]. However, the exact neural correlates of the different types of copying errors are currently unknown.

Copying can be assessed using a wide range of tasks, with some studies using the pentagon copying task of the MMSE [e.g., 13] and other studies using the Rey-Osterrieth complex figure [e.g., 3]. The pentagon copying task is particularly interesting, given the wide use of the MMSE to assess general cognitive abilities and the known time benefits of using short drawing tests early in the disease process [14]. Qualitative and reproducible methods to evaluate the pentagon sub-item have long been lacking, however. This has resulted in a debate in the literature about the criteria for considering a copy normal or abnormal [e.g., 15], casting doubt about the observed dissociations between the different neurodegenerative disorders based on the pentagon copying task. Recently, a qualitative scoring method for the pentagon copy test was developed with a good interrater and intrasubject reliability [16]. This method, termed the "qualitative scoring method for the pentagon copy test" (QSPT), categorizes different possible errors in copying, resulting in more information about the quality of the copy than the dichotomous categories "correct" or "incorrect." The use of dichotomous categories potentially omits relevant information on the cognitive processes associated with copying figures. This new approach is therefore promising, as specific errors on this copying test could point to specific underlying neural correlates. QSPT allows for scoring the pentagon drawing on various specific categories, such as the correct number of angles, the correct distance between the objects, and any possible incorrect rotation that might be present in the copy. For instance, Mitolo et al. [17] proposed that the number of angles is the optimal way to distinguish between dementia with Lewy bodies and AD [see also 6].

Currently, the different neural correlates of the individual categories of the QSPT are lacking. Therefore, to assess whether the different categories of errors on the pentagon drawing relate to different neural correlates, we examined the pentagon drawings in a memory clinic population, specifically persons with subjective cognitive complaints (SCC), 
Van der Stigchel et al.: Parietal Involvement in Constructional Apraxia

$\mathrm{MCI}$, or early dementia due to AD. More specifically, we correlated (regional) gray matter volumes on 3T MRI with performance on the different categories of the QSPT. Based on the previous literature, we expected errors in perseveration and planning to be associated with lower frontal gray matter volumes, whereas errors in the spatial relationship among constituent elements to be associated with lower parietal gray matter volumes.

\section{Methods}

Participants

Ninety-four participants with cognitive complaints were recruited through the memory clinic at the University Medical Center Utrecht between October 2009 and February 2013. Eligibility criteria for a standardized research workup for these patients were: (1) Mini-Mental Status Examination (MMSE) score $\geq 20$, (2) no other psychiatric or neurological disorder that could affect cognitive functioning apart from neurodegenerative disorders or cerebrovascular disease, (3) no recent nondisabling stroke ( $<2$ years) or any disabling stroke, major depression or a history of alcohol or substance abuse. The standardized evaluation included medical history, physical and neurological examination, laboratory testing (general measures, such as glucose, kidney functioning, hemoglobin, and TSH, which are commonly performed in a memory clinic setting), neuropsychological assessment (described later in more detail), and a 3T MRI scan. Four participants were excluded due to unreliable volumetric measurements on MRI (e.g., movement artefacts). For the present study, all participants with a diagnosis of early dementia due to AD (eAD), amnestic MCI (aMCI), or SCC were included. Diagnoses were established in a multidisciplinary meeting according to internationally accepted criteria (NINCDS-ADRDA criteria for AD [18]; Petersen criteria for aMCI, i.e. 1.5 SD below the normative average [19]).

Forty-one participants met the criteria for eAD (30 probable AD, 11 possible AD). Patients with other types of dementia $(n=4)$ were excluded. Thirty-eight participants met the criteria for aMCI. Participants with SCC $(n=7)$ were included if they had acquired SCC, without objective impairments on neuropsychological testing. For the purpose of this study, participants with SCC were included to ensure a wider total performance range on neuropsychological assessment and gray matter volumes on MRI. The study was carried out in accordance with the principles of the Declaration of Helsinki and approved by the local ethics committee. All participants gave written informed consent.

\section{Neuropsychological Assessment}

Participants performed a standardized neuropsychological assessment. Tests were administered by a trained neuropsychologist and covered the major cognitive functions. Briefly, the test battery included the following tests: the forward condition of the Digit Span (Wechsler Adult Intelligence Scale 3rd edition, WAISIII) was used to assess attention. Working memory was assessed with the backward condition of the WAIS-III Digit Span. For memory, the Visual Association Test and the Dutch version of the Rey Auditory Verbal Learning Test (RAVLT, immediate and delayed recall) were used. The Trail Making Test was included as a measure of processing speed (Part A) and executive functioning (Part B/Part A ratio). Similarly, the Stroop Color Word Test was used to assess processing speed (Card I and II) and executive functioning (Card III/Card II ratio). Executive functioning and language were additionally assessed using a Category Fluency test (animal naming). Visual perception was assessed with the Incomplete Letters and Position Discrimination subtests of the Visual Object and Space Perception battery (VOSP). The Mini Mental-State Examination (MMSE) was included as a measure of global cognition functioning.

\section{Reevaluation of MMSE-Pentagon Scoring}

Copying the overlapping pentagons is a standard sub-item of the MMSE. Traditionally, it is scored dichotomously as either correct or incorrect. A correct copy entails the reproduction of two figures with 5 angles that intersect forming a four-angled figure. Qualitative evaluation of the pentagon copy is generally not performed, thereby omitting potentially relevant information on the cognitive processes associated with copying figures. To this aim, Caffarra et al. [16] introduced a qualitative and reproducible method to evaluate the pentagon copy (qualitative scoring method of the pentagon copy test; QSPT). It takes into account five criteria of judgment (Table 1): number of angles, distance/intersection between the two figures, closing/ opening of the contour, rotation of one or both pentagons, and closing-in. A total score is also calculated corresponding to the sum of the individual scores of each parameter, ranging from 0 to 13 . All pentagon 
Van der Stigchel et al.: Parietal Involvement in Constructional Apraxia

Table 1. Qualitative scoring method of the pentagon copying test (QSPT) [16]

\begin{tabular}{|c|c|c|}
\hline Parameter & Performance & Score \\
\hline \multirow[t]{5}{*}{ 1. Number of angles } & 10 & 4 \\
\hline & $10 \pm 1$ & 3 \\
\hline & $10 \pm 2$ & 2 \\
\hline & $7-5$ & 1 \\
\hline & $<5$ or $>12$ & 0 \\
\hline \multirow[t]{5}{*}{ 2. Distance/intersection } & Correct intersection & 4 \\
\hline & Wrong intersection & 3 \\
\hline & Contact without intersection & 2 \\
\hline & No contact, distance $<1 \mathrm{~cm}$ & 1 \\
\hline & No contact, distance $>1 \mathrm{~cm}$ & 0 \\
\hline \multirow[t]{3}{*}{ 3. Closure/openinga } & Closing both figures & 2 \\
\hline & Closing only one figure & 1 \\
\hline & Opening both figures & 0 \\
\hline \multirow[t]{3}{*}{ 4. Rotation ${ }^{\mathrm{b}}$} & Correct orientation of both figures & 2 \\
\hline & $\begin{array}{l}\text { Rotation of one figure (either one figure is absent or it is not a } \\
\text { pentagon, then it is not assessable) }\end{array}$ & 1 \\
\hline & Rotation of both figures (or both not assessable like pentagons) & 0 \\
\hline \multirow[t]{2}{*}{ 5. Closing-in } & Absent & 1 \\
\hline & Present & 0 \\
\hline Total & Sum of $1-5$ & $0-13$ \\
\hline
\end{tabular}

Scoring criteria based on Caffarra et al. [16]. ${ }^{\text {a }}$ Figure is considered close even though two sides do not touch each other but the distance is $\leq 1 \mathrm{~mm}$. ${ }^{b}$ When there is no figure or figure is not a pentagon (then rotation is not assessable) the score is 0 . When rotation is less than $45^{\circ}$, figure is not considered rotated. Tremor is ignored.

drawings were collected from the original MMSE forms and were independently reevaluated according to the QSPT criteria by two of the authors (S.v.d.S., E.v.d.B.). Any discrepancies between the scores were solved in a consensus meeting. See scoring examples in Figure 1.

\section{MRI Dato}

MRI data were acquired on a Philips 3T scanner (Achieva; Philips, Best, The Netherlands). For each participant, a standardized MRI scan protocol was performed, consisting of a 3D T1-weighted sequence (192 slices, voxel size: $1.00 \times 1.00 \times 1.00 \mathrm{~mm}$, repetition time/echo time: 7.9/4.5 ms). The 3D T1-weighted images were processed using the recon-all pipeline in Freesurfer (http://surfer.nmr.mgh.harvard.edu [20]). Within this pipeline, the brain mask and reconstructed cortical surfaces were visually inspected, and segmentation inaccuracies were manually corrected according to the Freesurfer user guidelines. The Desikan-Killiany gyral-based cortical atlas [21] was used to subdivide the gray matter segmentations into anatomical/functional brain regions. The gray matter volumes per lobe and per brain region were extracted and used for further analyses.

\section{Statistical Analysis}

Between-group differences in characteristics were analyzed with analysis of variance for continuous variables, Mann-Whitney U tests for nonparametric data, and $\chi^{2}$ tests for proportions. The association between the total QSPT score and gray matter volume of the total frontal, parietal, temporal, and occipital lobes was examined in the total study population by linear regression analysis adjusted for age, sex, and total intracranial volume. In case of a statistically significant association, subsequent linear regression analysis was performed on the gray matter volume of the subareas of this lobe and the subscores of the QSPT (adjusted for age, sex, and total intracranial volume). As no participant showed closing-in on the pentagon copy, this variable was dropped from the analysis. 
Fig. 1. Scoring examples of the QSPT pentagon scoring system.

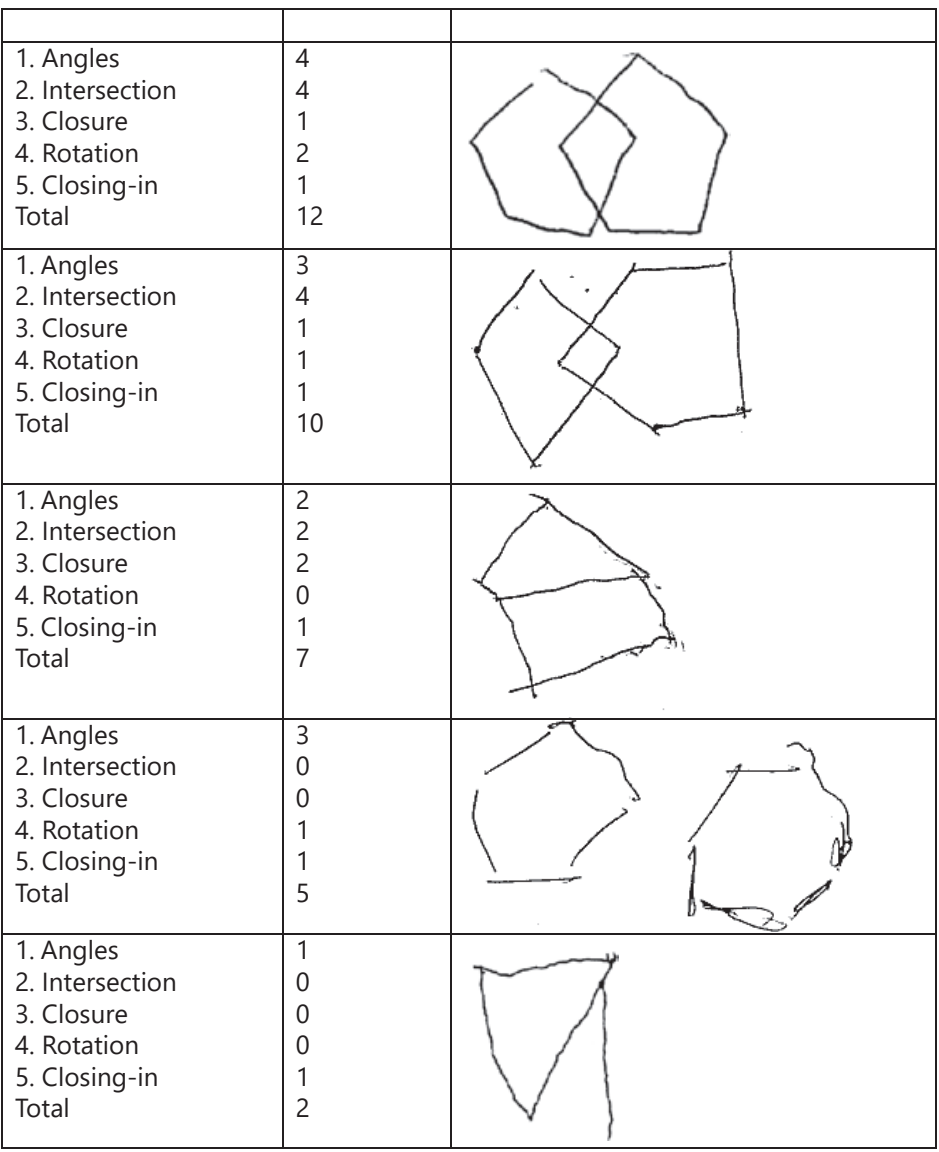

Further, a sensitivity analysis was performed on "classic" brain areas associated with Alzheimer type pathology (e.g., medial temporal lobe, precuneus) and measures of memory (Visual Association Test, RAVLT immediate and delayed recall). This way, we could determine whether the observed associations with the QSPT subscores are specific to constructional apraxia or due to a more general effect of disease severity. All analyses were performed with SPSS version 21 (IBM Corp.).

\section{Results}

The characteristics of the study population are presented in Table 2. The aMCI and eAD patients were older than the SCC group $(F(2,83)=2.17, p<0.05)$. The eAD group had a lower level of education $(p=0.03)$. There was no significant between-group difference in sex $\left(\chi^{2}(2)=5.0, p=0.08\right)$. As expected, the MMSE score was significantly lower for the eAD group compared to aMCI and SCC $(p<0.01)$. The mean MMSE score of 24.4 (SD 2.3) for the eAD patients indeed indicates early stage AD. The original dichotomous (correct/incorrect) scoring method of the pentagon copy results in a high percentage $(>78 \%)$ of correct responses. There was no significant between group difference in total QSPT score $(F(2,83)=1.49, p<$ $0.05)$.

Association between Gray Matter Volume and QSPT Pentagon Scoring

The QSPT total score showed a significant association with parietal gray matter volume (adjusted standardized regression coefficient beta 0.39 (95\% confidence interval 0.08 to 
Table 2. Characteristics of the study sample

\begin{tabular}{lccc}
\hline & SCC & MCI & AD \\
\hline Subjects, $n$ & 7 & 38 & 41 \\
Age, years & $69.3(8.4)$ & $75.1(8.6)$ & $76.4(8.2)$ \\
Males & $6(86 \%)$ & $16(42 \%)$ & $23(56 \%)$ \\
Education $^{\mathrm{a}}$ & $5.1(1.5)$ & $5.2(1.3)$ & $4.7(1.6)$ \\
Median CDR score (IQR) & $0.5(0-0.5)$ & $0.5(0.5-0.5)$ & $1(0.5-1.0)$ \\
MMSE & $28.9(0.7)$ & $27.4(1.8)$ & $24.4(2.3)$ \\
\hline Pentagon scoring & & & \\
Correct pentagon copy & $6(86 \%)$ & $34(90 \%)$ & $32(78 \%)$ \\
QSPT total (range 0-13) & $11.9(1.5)$ & $11.6(1.3)$ & $10.9(2.1)$ \\
\hline Gray matter volumes (in \% ICV) & & & $9.4(1.2)$ \\
Frontal GMV & $10.6(1.3)$ & $9.7(1.1)$ & $6.2(0.7)$ \\
Parietal GMV & $7.3(1.0)$ & $6.6(0.7)$ & $5.5(0.7)$ \\
Temporal GMV & $6.7(0.7)$ & $5.9(0.8)$ & $2.5(0.3)$ \\
Occipital GMV & $3.0(0.4)$ & $2.7(0.3)$ & \\
\hline
\end{tabular}

Data are means (SD) or $n(\%)$ unless otherwise specified. SCC, subjective cognitive complaints; AD, Alzheimer's disease, GMV, gray matter volume; CDR, Clinical Dementia Rating; IQR, interquartile range; MMSE, Mini-Mental State Examination; QSPT, qualitative scoring method for the pentagon copy test; MCI, mild cognitive impairment. ${ }^{\text {a }}$ Education scored in 7 levels according to the Dutch system, $1=$ less than primary school, 7 = university degree.

$0.71, p<0.01$ ), but not with the gray matter volumes of the frontal, temporal, or occipital lobes ( 0.28 [ -0.08 to 0.64 ]; 0.25 [ -0.06 to 0.57 ]; 0.25 [ -0.05 to 0.55 ], all $p>0.05$ ). Table 3 shows the results of the linear regression analysis of the gray matter volume of the cortical subregions of the parietal lobe and the subscores of the QSPT. These analyses show that the superior, inferior, supramarginal, and postcentral parietal gray matter volumes are associated with the number of angles and the intersection scores of the pentagon copy (range 0.27 [0.01 to 0.53] to 0.45 [0.19 to 0.72$], p<0.05$ ). In addition, the supramarginal parietal gray matter volume is associated with rotation of the pentagon ( 0.30 [0.02 to 0.58$], p<0.05)$, and the posterior cingulate gray matter volume shows an association with the intersection of the pentagon copy $(0.29$ [0.03 to 0.55$], p<0.05)$. The closure criterion of the QSPT was not associated with any of the parietal cortical subregions (range -0.17 [ -0.48 to 0.14 ] to 0.10 [ -0.16 to 0.36 ], all $p>0.05$ ). Because of the ordinal nature of several of the QSPT variables, all analyses were repeated with ordinal regression analysis (PLUM). These analyses yielded highly similar results (data not shown).

\section{Sensitivity Analyses}

The previously described results indicate an association between (cortical subregions of) the parietal lobe and the qualitative scoring criteria of the pentagon. As a sensitivity analysis, we additionally examined the association between brain regions that are typically affected in $\mathrm{AD}$ and measures of memory (Visual Association Test, RAVLT immediate and delayed recall). Indeed, the Visual Association Test is significantly associated with the volume of the precuneus (standardized regression coefficient 0.58 [0.30 to 0.88], $p<0.01$ ) and the middle temporal ( 0.32 [0.03 to 0.59 ], $p<0.05$ ) and parahippocampal volume (0.31 [0.05 to 0.53 ], $p<0.05$ ), but also shows modest associations with the inferior temporal and fusiform gyrus (see Table 4). The RAVLT delayed recall test also shows specific associations with the precuneus $(0.46[0.20$ to 0.73$], p<0.01$ ), the superior, middle, and inferior temporal regions, and the volumes of the 
Table 3. Linear regression analysis of the gray matter volume in parietal subregions and QSPT pentagon scoring

\begin{tabular}{|c|c|c|c|c|c|}
\hline & QSPT total & QSPT angles & QSPT intersection & QSPT closure & QSPT rotation \\
\hline Superior parietal & $0.32(0.07 \text { to } 0.57)^{*}$ & $0.31(0.06 \text { to } 0.55)^{*}$ & $0.31(0.06 \text { to } 0.56)^{*}$ & $0.10(-0.16$ to 0.36$)$ & $0.16(-0.09$ to 0.42$)$ \\
\hline Inferior parietal & $0.18(-0.09$ to 0.45$)$ & $0.27(0.01 \text { to } 0.53)^{*}$ & $0.28(0.02 \text { to } 0.55)^{*}$ & $-0.16(-0.44$ to 0.11$)$ & $0.10(-0.17$ to 0.37$)$ \\
\hline Supramarginal & $0.37(0.10 \text { to } 0.65)^{*}$ & $0.45(0.19 \text { to } 0.72)^{*}$ & $0.39(0.11 \text { to } 0.66)^{*}$ & $-0.07(-0.36$ to 0.22$)$ & $0.30(0.02 \text { to } 0.58)^{*}$ \\
\hline Postcentral & $0.28(-0.02$ to 0.57$)$ & $0.33(0.04 \text { to } 0.62)^{*}$ & $0.37(0.08 \text { to } 0.66)^{*}$ & $-0.12(-0.43$ to 0.18$)$ & $0.22(-0.07$ to 0.51$)$ \\
\hline Precuneus & $0.13(-0.17$ to 0.44$)$ & $0.25(-0.05$ to 0.55$)$ & $0.21(-0.09$ to 0.52$)$ & $-0.17(-0.48$ to 0.14$)$ & $0.10(-0.21$ to 0.40$)$ \\
\hline Posterior cingulate & $0.28(0.02 \text { to } 0.54)^{*}$ & $0.24(-0.02$ to 0.49$)$ & $0.29(0.03 \text { to } 0.55)^{*}$ & $0.08(-0.19$ to -0.35$)$ & $0.18(-0.08$ to 0.44$)$ \\
\hline Isthmus cingulate & $0.09(-0.20$ to 0.39$)$ & $0.20(-0.08$ to 0.49$)$ & $0.01(-0.29$ to 0.30$)$ & $0.04(-0.26$ to 0.34$)$ & $0.13(-0.16$ to 0.42$)$ \\
\hline
\end{tabular}

Analyses adjusted for age, sex, and total intracranial volume. Data are standardized regression coefficients beta and $95 \%$ confidence interval. QSPT, qualitative scoring method for the pentagon copy test. $* p<0.05$

Table 4. Linear regression analysis of the association between gray matter volume and memory performance

\begin{tabular}{lllr}
\hline & RAVLT immediate & RAVLT delayed & Visual association test \\
\hline Parietal gray matter volume & & & \\
Superior parietal & $0.23(-0.03$ to 0.50$)$ & $0.18(-0.05$ to 0.41$)$ & $0.08(-0.17$ to 0.33$)$ \\
Inferior parietal & $0.51(0.26 \text { to } 0.78)^{* *}$ & $0.26(0.02 \text { to } 0.50)^{*}$ & $0.24(-0.07$ to 0.50$)$ \\
Supramarginal & $0.42(0.15 \text { to } 0.71)^{* *}$ & $0.14(-0.12$ to 0.40$)$ & $-0.01(-0.29$ to 0.26$)$ \\
Postcentral & $0.37(0.08 \text { to } 0.68)^{*}$ & $0.21(-0.06$ to 0.48$)$ & $0.25(-0.03$ to 0.53$)$ \\
Precuneus & $0.58(0.30 \text { to } 0.88)^{* *}$ & $0.46(0.20 \text { to } 0.73)^{* *}$ & $0.57(0.30 \text { to } 0.53)^{* *}$ \\
Posterior cingulate & $0.15(-0.12$ to 0.42$)$ & $0.17(-0.07$ to 0.41$)$ & $0.10(-0.15$ to 0.35$)$ \\
Isthmus cingulate & $0.19(-0.11$ to 0.49$)$ & $0.14(-0.12$ to 0.41$)$ & $0.16(-0.12$ to 0.44$)$ \\
\hline Temporal gray matter volume & & & \\
Superior temporal & $0.59(0.03 \text { to } 0.86)^{* *}$ & $0.30(0.03 \text { to } 0.57)^{*}$ & $0.22(-0.07$ to 0.49$)$ \\
Middle temporal & $0.50(0.22 \text { to } 0.80)^{* *}$ & $0.36(0.09 \text { to } 0.64)^{*}$ & $0.32(0.03 \text { to } 0.59)^{*}$ \\
Inferior temporal & $0.42(0.13 \text { to } 0.68)^{* *}$ & $0.40(0.13 \text { to } 0.64)^{* *}$ & $0.30(0.02 \text { to } 0.54)^{*}$ \\
Fusiform & $0.38(0.12 \text { to } 0.67)^{* *}$ & $0.18(-0.07$ to 0.45$)$ & $0.27(0.01 \text { to }-0.54)^{*}$ \\
Transverse temporal & $0.25(0.01 \text { to } 0.49)^{*}$ & $0.15(-0.07$ to 0.38$)$ & $0.18(-0.05$ to 0.40$)$ \\
Entorhinal & $0.17(-0.06$ to 0.40$)$ & $0.22(0.01 \text { to } 0.43)^{*}$ & $0.09(-0.08$ to 0.27$)$ \\
Parahippocampal & $0.49(0.22 \text { to } 0.73)^{* *}$ & $0.45(0.22 \text { to } 0.68)^{* *}$ & $0.31(0.05 \text { to } 0.53)^{*}$ \\
\hline
\end{tabular}

Analyses adjusted for age, sex, and total intracranial volume. Data are standardized regression coefficients beta and $95 \%$ confidence interval. RAVLT, Rey Auditory Verbal Learning Test. ${ }^{*} p<0.05,{ }^{* *} p<0.01$.

entorhinal and parahippocampal regions (range of standardized regression coefficients 0.22 [0.01 to 0.43 ] to 0.40 [0.13 to 0.64$]$, all $p<0.05$ ). The RAVLT immediate recall test showed association with a wider range of both parietal and temporal areas (Table 4), possibly indicating additional involvement of other (nonmemory) processes, such as attention and executive functioning.

\section{Discussion}

Deficits in copying can occur after both frontal and parietal damage. The aim of the present study was to correlate the different categories of errors on the pentagon drawing to neural correlates. We used a novel method to categorize different possible errors in copying, the QSPT [16].

Results showed that the total score of the QSPT was specifically associated with parietal gray matter volume and not with frontal, temporal, and occipital gray matter volume. Results 
from overall performance on the pentagon task have until now been inconsistent (and focused specifically on patients with Parkinson's disease), with one study pointing specifically to gray matter volume in the frontal cortex [22] and another study revealing additional temporoparietal involvement [23]. Importantly, these studies did not look at the different categories of errors. We included patients with different neural disorders and/or cognitive complaints to ensure a wide range of gray matter volumes on MRI.

The QSPT provides a fine-grained categorization of errors. When examining the correlation of the different categories with gray matter volume, we only observed clear associations with parietal regions. Although the closure criterion was not associated with any region, the number of angles and the intersection score were associated with various subregions of the parietal cortex. The rotation score was the only category that was associated with one parietal region, namely the supramarginal parietal cortex. The validity of these results was supported by a sensitivity analysis in which we observed an association between brain regions that are typically affected in $\mathrm{AD}$ and measures of memory, indicating that the observed association with the QSPT subscores are specific to constructional apraxia and not due to a more general effect of disease severity.

Of the various categories of errors, the correct number of angles is of particular interest. Previous studies using the QSPT have observed that the number of angles is able to distinguish dementia with Lewy bodies from AD [6,17]. Mitolo et al. [17] claim that correct copying of the number of errors requires subjects to generate a visual image of the image stored in semantic memory. Although this is an interesting suggestion, it is difficult to understand how such a mechanism would be specific to the number of angles and not be reflected in a correct intersection or a correct closure. Furthermore, our study has revealed that performance on the correct number of angles has the same neural correlates as the intersection score.

Given the strong involvement of the posterior parietal cortex in accounting for the different categories of errors, one interesting hypothesis to explain the copying deficits observed in our study was proposed by Russell et al. [3]. They proposed that constructional apraxia can be attributed to the failure to integrate visual information correctly from one fixation to the next, a process called "spatial remapping." To process visual information, the visual system redirects the high-acuity fovea to objects of interest in the visual field by rapidly moving the eyes, i.e. saccades. Although saccades dramatically alter the visual input, we subjectively experience a stable visual world, that is, a world in which items of interest are available for processing both before and after a saccade [24, 25]. Therefore, with each new fixation, our brain has to integrate the old and new retinal images with information about the current eye position and the magnitude and direction in which gaze was displaced by the most recent saccade. The saving, updating, and relocalization of the different parts of a visual scene, referred to as spatial remapping, allows us to accurately determine the location of external targets and generate eye or limb movements to these targets [26,27]. This process has been particularly linked to the posterior parietal cortex [28]. After damage to the posterior parietal cortex, patients have difficulties in spatial remapping $[3,29,30]$, which might result in difficulties on a copying task. In such a task, spatial information about a figure has to be maintained across saccades, as copying requires the execution of saccades between the original figure and the participant's drawing. When spatial information is lost across saccades, the construction of the original figure is lost, resulting in a loss of memory for the correct number of angles and the correct rotation. Furthermore, a loss of the relation between the two individual pentagons across saccades will result in an incorrect intersection between the two figures. The only category that was not specific to a neural region, the closure criterion, might then only require object memory. Good performance on this criterion only involves memory that the objects need to be closed, and no spatial information. 
Although we aimed to include a wide range of gray matter atrophy by including persons with SCC, MCI, and early dementia due to AD, it might be that the current findings are specific to the current sample. Most importantly, we included AD patients in a relatively early stage of their disease. This could have influenced the fact that we only observed associations with parietal areas. For instance, we excluded one category of errors, namely closing-in, as these errors were not observed in our sample. Interestingly, errors in closing-in have been associated with frontal dysfunction [e.g., 31], although these errors of closing-in were not observed in the pentagon copying task but on other, more elaborate, copying tasks. As our study was specific to the relatively simple pentagon copying task, our results might not generalize to tasks tapping into more complex visuoconstruction, such as the Rey Complex Figure Task. Further, our groups were not equally large, with only 7 patients with SCC, compared to the much larger groups of patients with $\mathrm{MCI}$ and early dementia due to AD (both around 40). It is therefore important to note that our study was not aimed at comparing performance between these groups, but rather focused on the neural correlates of the different types of copying errors. Finally, our diagnoses of AD were not supported by biomarker evidence of Alzheimer's pathology.

As mentioned earlier, constructional apraxia is not a uniform deficit. The current study has revealed that the pentagon copying task is specifically sensitive to parietal damage and might therefore be a too simple task to grasp the full spectrum of possible drawing errors as a result of neurodegenerative gray matter atrophy. We have shown that a more fine-grained analysis of the errors in this task reveals that the intersection score and the number of angles share their underlying neural correlates and are associated with specific subregions of the parietal cortex.

\section{Appendix}

Members of the Utrecht Vascular Cognitive Impairment (VCI) Study Group involved in the present study (in alphabetical order by department).

University Medical Center Utrecht, the Netherlands, Department of Neurology: G.J. Biessels, C. Maher, S.M. Heringa, L.J. Kappelle, Y.D. Reijmer, J. Verwer; Department of Radiology/Image Sciences Institute: J. de Bresser; Department of Geriatrics: H.L. Koek; Hospital Diakonessenhuis Zeist, the Netherlands: M. Hamaker, R. Faaij, M. Pleizier, E. Vriens; Erasmus MC University Medical Center, the Netherlands, Department of Neurology: E. van den Berg.

\section{Disclosure Statement}

There are no conflicts of interest.

\section{References}

1 Guérin F, Ska B, Belleville S: Cognitive processing of drawing abilities. Brain Cogn 1999;40:464-478.

2 Hier DB, Mondlock J, Caplan LR: Recovery of behavioral abnormalities after right hemisphere stroke. Neurology 1983;33:345-350.

3 Russell C, Deidda C, Malhotra P, Crinion JT, Merola S, Husain M: A deficit of spatial remapping in constructional apraxia after right-hemisphere stroke. Brain 2010;133:1239-1251.

4 Ala TA, Hughes LF, Kyrouac GA, Ghobrial MW, Elble RJ: Pentagon copying is more impaired in dementia with Lewy bodies than in Alzheimer's disease. J Neurol Neurosurg Psychiatry 2001;70:483-488.

5 Parsey CM, Schmitter-Edgecombe M: Quantitative and qualitative analyses of the clock drawing test in mild cognitive impairment and Alzheimer disease: evaluation of a modified scoring system. J Geriatr Psychiatry Neurol 2011;24:108-118. 
Van der Stigchel et al.: Parietal Involvement in Constructional Apraxia

6 Cagnin A, Bussè C, Jelcic N, Gnoato F, Mitolo M, Caffarra P: High specificity of MMSE pentagon scoring for diagnosis of prodromal dementia with Lewy bodies. Parkinsonism Relat Disord 2015;21:303-305.

7 Kleist K: Gehirnpathologie. Leipzig, Barth, 1934.

8 Trojano L, Grossi D, Flash T: Visuospatial and visuoconstructive deficits; in Goldenberg G, Miller B (eds): Handbook of Clinical Neurology. Amsterdam, Elsevier, 2008, pp 373-392.

9 Farah M: Disorders of visuo-spatial perception and cognition; in Heilman KM, Valenstein E (eds): Clinical Neuropsychology. New York, Oxford University Press, 2003, pp 146-160.

10 Trojano L, Gainotti G: Drawing disorders in Alzheimer's disease and other forms of dementia. J Alzheimer Dis 2016;53:31-52.

11 Thompson JC, Stopford CL, Snowden JS, Neary D: Qualitative neuropsychological performance characteristics in frontotemporal dementia and Alzheimer's disease. J Neurol Neurosurg Psychiatry 2005;76:920-927.

12 Molteni F, Traficante D, Ferri F, Isella V: Cognitive profile of patients with rotated drawing at copy or recall: a controlled group study. Brain Cogn 2014;85:286-290.

13 Cormack F, Aarsland D, Ballard C, Tovée MJ: Pentagon drawing and neuropsychological performance in Dementia with Lewy Bodies, Alzheimer's disease, Parkinson's disease and Parkinson's disease with dementia. Int J Geriatr Psychiatry 2004;19:371-377.

14 Ericsson K, Forssell L G, Holmén K, Viitanen M, Winblad B: Copying and handwriting ability in the screening of cognitive dysfunction in old age. Arch Gerontol Geriatr 1996;22:103-121.

15 Della Sala S, Turnbull 0, Beschin N, Perini M: Orientation agnosia in pentagon copying. J Neurol Neurosurg Psychiatry 2002;72:129-130.

16 Caffarra P, Gardini S, Dieci F, Copelli S, Maset L, Concari L, et al: The qualitative scoring MMSE pentagon test (QSPT): a new method for differentiating dementia with Lewy body from Alzheimer's disease. Behav Neurol 2013;27:213-220.

17 Mitolo M, Salmon D P, Gardini S, Galasko D, Grossi E, Caffarra P: The new Qualitative Scoring MMSE Pentagon Test (QSPT) as a valid screening tool between autopsy-confirmed dementia with Lewy bodies and Alzheimer's disease. J Alzheimer Dis 2014;39:823-832.

18 McKhann G, Drachman D, Folstein M, Katzman R, Price D, Stadlan EM: Clinical diagnosis of Alzheimer's disease: report of the NINCDS-ADRDA Work Group under the auspices of Department of Health and Human Services Task Force on Alzheimer's Disease. Neurology 1984;34:939-994.

19 Petersen RC, Smith GE, Waring SC, Ivnik RJ, Tangalos EG, Kokmen E: Mild cognitive impairment: clinical characterization and outcome. Arch Neurol 1999;56:303-308.

20 Fischl B: FreeSurfer. Neuroimage 2012;62:774-781.

21 Desikan RS, Ségonne F, Fischl B, Quinn BT, Dickerson BC, Blacker D, et al: An automated labeling system for subdividing the human cerebral cortex on MRI scans into gyral based regions of interest. Neuroimage 2006; 31:968-980.

22 Filoteo JV, Reed JD, Litvan I, Harrington DL: Volumetric correlates of cognitive functioning in nondemented patients with Parkinson's disease. Mov Disord 2014;29:360-367.

23 Garcia-Diaz AI, Segura B, Baggio HC, Marti MJ, Valldeoriola F, Compta Y, et al: Structural MRI correlates of the MMSE and pentagon copying test in Parkinson's disease. Parkinsonism Relat Disord 2014;20:1405-1410.

24 Fabius JH, Fracasso A, Van der Stigchel S: Spatiotopic updating facilitates perception immediately after saccades. Sci Rep 2016;6:1-11.

25 Zerr P, Gayet S, Mulder K, Pinto Y, Sligte I, Van der Stigchel S: Remapping high-capacity, pre-attentive, fragile sensory memory. Sci Rep 2017;7:15940.

26 Van der Stigchel S, Hollingworth A: Visuospatial working memory as a fundamental component of the eye movement system. Curr Dir Psychol Sci 2018;27:136-143.

27 Schut MJ, Van der Stoep N, Postma A, Van der Stigchel S: The cost of making an eye movement: a direct link between visual working memory and saccade execution. J Vis 2017;17:15.

28 Duhamel J-R, Colby CL, Goldberg ME: The updating of the representation of visual space in parietal cortex by intended eye movements. Science 1992;255:90-92.

29 Pisella L, Alahyane N, Blangero A, Thery F, Blanc S, Pelisson D: Right-hemispheric dominance for visual remapping in humans. Philos Trans R Soc Lond B Biol Sci 2011;366:572-585.

30 Pisella L, Mattingley JB: The contribution of spatial remapping impairments to unilateral visual neglect. Neurosci Biobehav Rev 2004;28:181-200.

31 De Lucia N, Trojano L, Vitale C, Grossi D, Barone P, Santangelo G: The closing-in phenomenon in Parkinson's disease. Parkinsonism Relat Disord 2015;21:793-796. 